Research Paper

\title{
Differences of Circulating Inflammatory Markers be- tween Large- and Small Vessel Disease in Patients with Acute Ischemic Stroke
}

Lili Zeng1,2\#, Xiaosong He ${ }^{2,3 \#}$, Jianrong Liu ${ }^{1}$, Ling Wang11, Suiqing Weng ${ }^{1}$, Yongting Wang ${ }^{2,3}$, Shengdi Chen ${ }^{1}$ and Guo-Yuan Yang1,2,3凶

1. Department of Neurology, Ruijin Hospital, School of Medicine

2. Neuroscience and Neuroengineering Center, Med-X Research Institute

3. School of Biomedical Engineering, Shanghai Jiao Tong University, Shanghai 200030, China

\# These authors contributed equally to this work.

$\triangle$ Corresponding author: Guo-Yuan Yang, MD, PhD, Neuroscience and Neuroengineering Center, Med-X Research Institute, Shanghai Jiao Tong University, 1954 Hua Shan Road, Shanghai 200030, China. Tel: 86-21-62933186 Fax: 86-21-62932302; E-mail:gyyang0626@gmail.com

() Ivyspring International Publisher. This is an open-access article distributed under the terms of the Creative Commons License (http://creativecommons.org/ licenses/by-nc-nd/3.0/). Reproduction is permitted for personal, noncommercial use, provided that the article is in whole, unmodified, and properly cited.

Received: 2013.05.08; Accepted: 2013.08.12; Published: 2013.08.22

\begin{abstract}
Background: The difference of inflammatory response between the pathogenesis of cerebral large- and small vessel disease after stroke remains unclear. In present study, we aim to determine the association of circulating inflammatory markers with different stroke subtype.

Methods: 99 patients with non-cardioembolic stroke were divided into large artery atherosclerosis (LAA) and small-artery occlusion (SAO) according to TOAST classification. A panel of plasma inflammatory markers including leukocyte, lymphocyte, CRP, fibrinogen, D-dimer, CD40L, IFN-y, IL-I $\alpha$, IL- I I, IL-6, IL-8, IL- I 7 and TNF- $\alpha$ were measured within 72 hours following cerebral ischemia. The relation of their levels in plasma with stroke subtype was further studied. All statistical data analysis was performed by SPSS 17.0 software.

Results: We found that only CRP were closely associated with stroke subtype $(p<0.05)$. Compared to SAO subgroup, the plasma levels of CRP was higher in LAA subgroup $(p<0.05)$. The predictive efficiency of CRP more than 3.2 for LAA was $85.7 \%$ sensitivity. The influencing factor of CRP includes IL-6, lymphocyte, fibrinogen and D-dimer.

Conclusion: LAA had a stronger activation of inflammation than SAO in the pathogenesis, which was associated with the changes of CRP.
\end{abstract}

Key words: CRP; Cytokine; Inflammation; Stroke subtype

\section{INTRODUCTION}

Inflammatory response plays an important role in the pathophysiology of acute ischemic stroke [1]. It is involved in the occurrence and development of cerebral ischemia. A higher plasma level of several inflammatory mediators is found in stroke patients compared to the healthy controls [2-4]. However, the difference of inflammatory response in stroke subtypes remains unclear [5-9]. Currently, the Trial of
Org 10172 in Acute Stroke Treatment (TOAST) criteria is the most widely used stroke classification to classify stroke etiology into five subtypes: large artery atherosclerosis (LAA), cardioembolic stroke (CE), small artery occlusion (SAO), other determined etiology and undetermined etiology. The classification is mainly based on electrocardiogram, Echocardiogram, cervical Doppler and MRI etc [10]. The LAA subtype 
results in stenosis or occlusion of intra or extracranial large arteries, the instable plaque, artery to artery embolism and hypo-perfusion. The lipohyalinosis, vasoconstriction, arteriosclerosis, and atheroma were suggested the cause of SAO $[8,10]$. The common pathophysiology of LAA and SAO was atherosclerosis and focal inflammation [9]. It is noted that LAA subtype has a higher recurrent risk and a worse clinical outcome than SAO subtype. It is necessary to determinate the difference of the pathogenesis between LAA and SAO, which was useful for the diagnosis and therapy in non-embolic stroke. In present study, we focus on the inflammatory markers in the difference between LAA and SAO. We choose a panel of biomarkers related to atherosclerosis and inflammation to measure, including leukocyte, lymphocyte, monocyte, CRP, fibrinogen, D-dimer, CD40L, IFN- $\gamma$, IL-1a, IL-1 $\beta$, IL-6, IL-8, IL-17 and TNF- $\alpha$, and further investigate their relation with non cardioembolic stroke subtype.

\section{MATERIALS AND METHODS}

\section{Study subjects}

Patients with acute non-cardioembolic ischemic stroke were recruited between September 2009 and October 2012, from the Department of Neurology, Ruijin Hospital, Shanghai Jiao Tong University, China. Two neurologists make a diagnosis of ischemic stroke based on World Health Organization criteria. The NIHSS score was evaluated during the first day of admission in hospital. The etiological diagnosis was done using TOAST classification criteria. All diagnosis was confirmed by MRI imaging (DWI). The infarct volume was calculated by ABC/ 2 methods [11]. Tumors, abnormal renal or liver function (plasma creatinine $>140 \mu \mathrm{mol} / \mathrm{L}$ or GPT $>75 \mathrm{IU} / \mathrm{L}$ or GOT $>40$ $\mathrm{U} / \mathrm{L}$ ), infectious diseases, immunity diseases, blood disorders, and recurrent stroke were excluded. The therapy protocol was based on the guidelines for stroke management in China. The blood tests (leukocyte, lymphocyte and monocyte) and lipid panel screen including cholesterol, triglyceride, LDL, HDL, ApoA, ApoB and Lp (a) were routinely measured in the laboratory of Ruijin hospital.

The study was approved by the Institutional Review Board of Shanghai JiaoTong University. Written informed consent was obtained from patients prior to study enrollment.

\section{Blood sample collection}

On the day of admission, $4 \mathrm{ml}$ blood sample was collected into an EDTA containing tube within 72 hours following cerebral ischemia. Blood sample was centrifuged and fractionated at $3000 \mathrm{~g}$ for 15 minutes at $4^{\circ} \mathrm{C}$. The plasma layer was stored at $-80^{\circ} \mathrm{C}$ for the measurement of individual biomarkers.

\section{Measurement of plasma inflammatory bi- omarkers}

Plasma pro-inflammatory cytokines including CD40L, IFN- $\gamma$, IL-1 $\alpha$, IL-1 $\beta$, IL-6, IL-8, IL-17 and TNF- $\alpha$ were measured by ELISA assay using Mosaic $^{\mathrm{TM}}$ ELISA human cytokine panel 1 Kit (R\&D Systems, Minneapolis, MN) according the protocol as described. The minimum detectable doses (MDD) were: CD40L $2.23 \mathrm{pg} / \mathrm{ml}$, IFN- $\gamma 1.38 \mathrm{pg} / \mathrm{ml}$, IL-1a 0.59 $\mathrm{pg} / \mathrm{ml}$, IL-1 $\beta 0.20 \mathrm{pg} / \mathrm{ml}$, IL-6 $0.41 \mathrm{pg} / \mathrm{ml}$, IL-8 0.59 $\mathrm{pg} / \mathrm{ml}, \mathrm{IL}-170.17 \mathrm{pg} / \mathrm{ml}$ and TNFa $1.65 \mathrm{pg} / \mathrm{ml}$. The percent coefficient of intra-assay and inter-assay variation was less than $8 \%$ and $10 \%$, respectively. Fibrinogen (Fg), D-dimer and CRP were measured by commercial ELISA Kit according the protocol (EIAab, Wuhan, China). For each standard and sample, duplicate readings were performed. The minimum detectable doses (MDD) were: Fg $0.01 \mathrm{~g} / \mathrm{L}$, D-dimer 0.01 $\mathrm{mg} / \mathrm{L}$ and CRP $0,039 \mathrm{mg} / \mathrm{L}$. The percent mean coefficient of intra-assay and inter-assay variation was within $8 \%$ and $10 \%$, respectively.

\section{Statistical analysis}

The statistical analysis of data was performed by SPSS 17.0 software. First, Kolmogorov- Smirnov test was used to determine the normal distribution or not for all data. Data with a non-normal distribution was presented as median (lower Quartile, upper Quartile). Nonparametric Mann Whitney U test was applied to study the relation between variables in LAA and SAO. Receiver operator characteristic (ROC) curve analysis was done for the determination of the predictive sensitivity and specificity of biomarker. The significant statistical value was considered as $p<0.05$.

\section{RESULTS}

\section{General information}

Ninety-nine patients with non cardioembolic stroke were recruited. According to TOAST, patients were further classified into LAA group $(n=52)$ and SAO group $(n=47)$. There is no statistically significant difference in age, sex, or risk factor, including a history of diabetes, hypertension or hypercholesterolemia (Table 1, $p>0.05$ ). But LAA group had a higher NIHSS score and infarct volume, compared to the SAO group (Table $1, p<0.05$ ).

\section{Lipid variable has no correlation with noncar- dioembolic stroke subtype.}

Among the panel of serum lipid examined, we did not find a significant association between lipid variables and noncardioembolic stroke subtype (Ta- 
ble 1, $p>0.05)$. Plasma cholesterol, triglyceride, LDL, HDL, ApoA, ApoB and Lp (a) showed a similar level in patients with LAA with that in patients with SAO (Table 1, $p>0.05$ ).

\section{None pro-inflammatory cytokine was related to noncardioembolic stroke subtype.}

Among the panel of circulating pro-inflammatory cytokines examined, we did not find a significant association between proinflammatory cytokines and noncardioembolic stroke subtype (Table 1, $p>0.05$ ). Plasma CD40L, IFN- $\gamma$, IL-1 $\alpha$, IL-1 $\beta$, IL-6, IL-8, IL-17 and TNF- $\alpha$ showed a similar level in patients with LAA with that in patients with SAO (Table 1, $p>0.05$ ).

\section{Fg and D-dimer were not associated with noncardioembolic stroke subtype.}

Among the panel of coagulation/fibrinolysis markers examined, we did not find a significant association between Fg, D-dimer and noncardioembolic stroke subtype (Table 1, $p>0.05$ ). Plasma Fg and D-dimer showed a similar level in patients with LAA with that in patients with SAO [3.25 $(2.53,4.08)$ vs. $3.10(2.70,3.60), p=0.40 ; 0.30(0.16,0.55)$ vs. $0.27(0.19$, $0.51), p=0.83$; respectively].

Table I Clinical characterizations and circulating inflammatory markers between large- and small vessel disease in patients with acute ischemic stroke.

\begin{tabular}{|c|c|c|c|}
\hline & $\begin{array}{l}\text { LAA } \\
(n=52)\end{array}$ & $\begin{array}{l}\text { SAO } \\
(n=47)\end{array}$ & Pvalue \\
\hline Race (Asian) & $100 \%$ & $100 \%$ & 1 \\
\hline Ethnicity (Han) & $100 \%$ & $100 \%$ & 1 \\
\hline Age (years) & $64(57,77)$ & $69(57,78)$ & 0.42 \\
\hline Gender(M/F) & $32 / 20$ & $29 / 18$ & 0.87 \\
\hline Hypertension & $84.6 \%$ & $78.7 \%$ & 0.12 \\
\hline Diabetes & $19.23 \%$ & $19.14 \%$ & 0.75 \\
\hline Dyslipidemia & $53.84 \%$ & $51.03 \%$ & 0.79 \\
\hline NIHSS & $5(3,10)$ & $2(1,3)$ & 0.001 \\
\hline Infarct volume & $3.97(1.3,27.80)$ & $0.45(0.16,1.82)$ & 0.001 \\
\hline WBC $\left(\times 10^{9} / \mathrm{L}\right)$ & $7.30(5.80,8.10)$ & $6.60(5.70,8.13)$ & 0.24 \\
\hline Lymphocyte $\left(\times 10^{9} / \mathrm{L}\right)$ & $1.79(1.20,2.18)$ & $1.70(1.32,2.19)$ & 0.80 \\
\hline Monocyte $\left(\times 10^{9} / \mathrm{L}\right)$ & $0.47(0.39,0.61)$ & $0.53(0.42,0.70)$ & 0.16 \\
\hline Cholesterol (mmol/L) & $4.81(4.14,5.60)$ & $4.78(4.10,5.67)$ & 0.80 \\
\hline Triglyceride $(\mathrm{mmol} / \mathrm{L})$ & $1.79(1.19,2.16)$ & $1.50(1.03,2.07)$ & 0.24 \\
\hline LDL (mmol/L) & $2.93(2.29,3.55)$ & $3.03(2.38,3.65)$ & 0.67 \\
\hline HDL (mmol/L) & $1.05(0.90,1.19)$ & $1.14(0.95,1.33)$ & 0.08 \\
\hline ApoA (mmol/L) & $1.21(1.05,1.40)$ & $1.17(1.04,1.46)$ & 0.72 \\
\hline ApoB (mmol/L) & $1.06(0.84,1.23)$ & $1.03(0.86,1.16)$ & 0.93 \\
\hline $\mathrm{Lp}(\mathrm{a})(\mathrm{mmol} / \mathrm{L})$ & $0.15(0.07,0.26)$ & $0.15(0.10,0.26)$ & 0.72 \\
\hline CD40L (pg/ML) & $139.66(91.29,238.46)$ & $139.30(90.35,269.80)$ & 0.74 \\
\hline 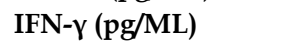 & $0(0,0)$ & $0(0,0)$ & 0.61 \\
\hline IL-1a (pg/ML) & $0(0,0)$ & $0(0,0)$ & 1 \\
\hline IL-1 $\beta$ (pg/ML) & $0(0,0)$ & $0(0,0)$ & 1 \\
\hline IL-6 (pg/ML) & $0.65(0.20,1.95)$ & $0.65(0.00,1.45)$ & 0.55 \\
\hline IL-8 (pg/ML) & $5.03(3.50,7.09)$ & $4.20(3.26,6.85)$ & 0.45 \\
\hline IL-17 (pg/ML) & $0(0,0)$ & $0(0,0)$ & 0.61 \\
\hline TNFa (pg/ML) & $6.93(4.48,9.34)$ & $6.10(4.56,8.70)$ & 0.46 \\
\hline $\mathrm{Fg}(\mathrm{g} / \mathrm{L})$ & $3.25(2.53,4.08)$ & $3.10(2.70,3.60)$ & 0.40 \\
\hline D-dimer (mg/L) & $0.30(0.16,0.55)$ & $0.27(0.19,0.51)$ & 0.83 \\
\hline CRP (mg/L) & $6.20(3.80,11.50)$ & $3.35(1.75,6.10)$ & 0.01 \\
\hline
\end{tabular}




\section{CRP was the only inflammatory mediator, which correlated to noncardioembolic stroke subtype.}

Among the panel of inflammatory mediators examined, CRP was the only one, which was closely associated with noncardioembolic stroke subtype (Table 1, $p<0.05$ ). Plasma CRP was significantly higher in patients with LAA subtype than in patients with SAO subtype $[6.20(3.80,11.50)$ vs. 3.35 (1.75, 6.10), $p=0.01$, Figure 1A]. ROC analysis showed that the optimal cut-off point of CRP was 3.20 [AUC 0.70 $(0.56,0.84), p=0.022]$, suggesting that a higher level of CRP more than 3.2 is associated with LAA subtype (Figure 1B, sensitivity $85.7 \%$ and specificity $48.1 \%$ ). The study about the time course of CRP in the acute stage after stroke showed that plasma CRP was sig- nificantly higher in patients with LAA subtype than in patients with SAO subtype within one day of onset (Table 2, $p<0.05$ ), but not within 1-3 days (Table 3, $p>0.05)$. The further study demonstrated that influencing factors correlated with CRP include IL-6, lymphocyte, fibrinogen, D-dimer and infarct volume but not NIHSS (Table $4, p<0.05$ ). In our study, the infarct volume of LAA and SAO was $3.97(1.3,27.80)$ and $0.45(0.16,1.82)$, respectively. We selected the overlap of the infarct volume (1.3-1.82) between the two groups to study the different inflammatory mediator levels between smaller large vessels stroke $(n=23)$ and SAO $(n=15)$. We found that smaller large vessels stroke had a higher CRP level than SAO (Table $5, p=0.05$ ).
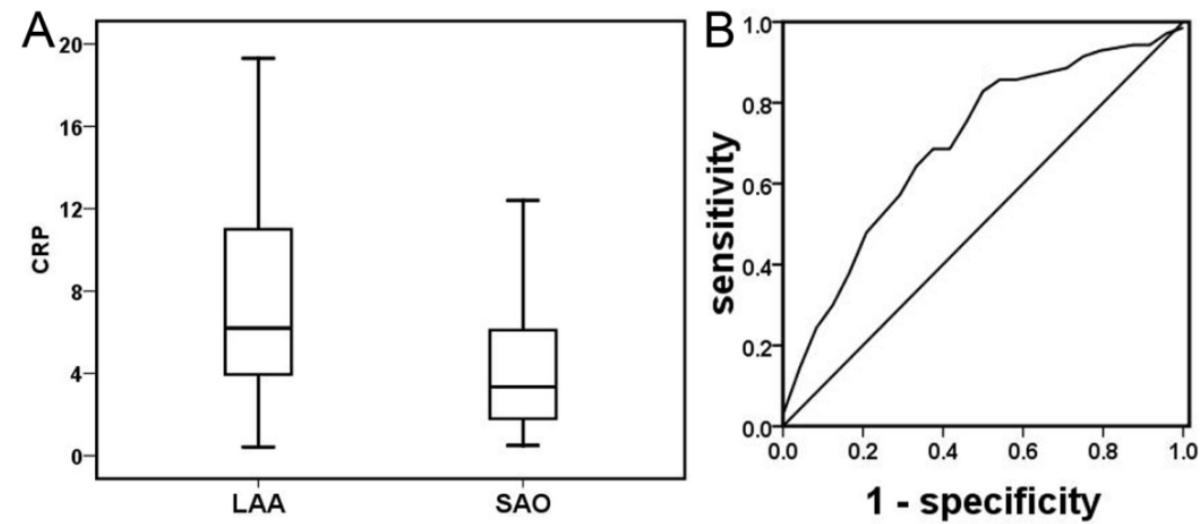

Figure I Plasma CRP in acute ischemia stroke patients with LAA subtype and SAO subtype. Compared to SAO subtype, plasma CRP was higher than LAA subtype in patients with acute ischemic stroke (Figure IA, $p<0.05$ ). ROC analysis of CRP demonstrated that its predicted efficiency for stroke LAA subtype was $85.7 \%$ sensitivity and $48.1 \%$ specificity [Figure IB, AUC $0.70(0.56,0.84), p=0.022$ ].

Table 2 Circulating inflammatory markers between large- and small vessel disease in patients with acute ischemic stroke within I day of onset.

\begin{tabular}{|c|c|c|c|}
\hline & $\begin{array}{l}\text { LAA } \\
(n=24)\end{array}$ & $\begin{array}{l}\text { SAO } \\
(n=18)\end{array}$ & P value \\
\hline WBC $\left(\times 10^{9} / \mathrm{L}\right)$ & $7.60(6.40,8.30)$ & $6.60(5.65,8.20)$ & 0.169 \\
\hline Lymphocyte $\left(\times 10^{9} / \mathrm{L}\right)$ & $1.69(1.07,2.13)$ & $1.73(1.375,2.505)$ & 0.280 \\
\hline Monocyte $\left(\times 10^{9} / \mathrm{L}\right)$ & $0.49(0.42,0.67)$ & $0.51(0.40,0.78)$ & 0.396 \\
\hline CD40L (pg/ML) & $125.70(88.09,275.28)$ & $240.48(133.81,378.00)$ & 0.057 \\
\hline 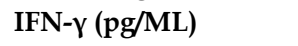 & $0(0,0)$ & $0(0,0)$ & 1 \\
\hline IL-1a (pg/ML) & $0(0,0)$ & $0(0,0)$ & 1 \\
\hline $\mathrm{IL}-1 \beta(\mathrm{pg} / \mathrm{ML})$ & $0(0,0)$ & $0(0,0)$ & 0.098 \\
\hline IL-6 (pg/ML) & $1.05(0.60,2.04)$ & $1.27(0,2.49)$ & 0.692 \\
\hline IL-8 (pg/ML) & $5.43(3.08,8.38)$ & $5.56(3.34,8.11)$ & 0.819 \\
\hline IL-17 (pg/ML) & $0(0,0)$ & $0(0,0)$ & 0.195 \\
\hline TNFa (pg/ML) & $6.33(4.32,9.65)$ & $5.11(4.40,7.46)$ & 0.416 \\
\hline $\mathrm{Fg}(\mathrm{g} / \mathrm{L})$ & $3.50(2.80,4.40)$ & $3.10(2.20,3.60)$ & 0.417 \\
\hline D-dimer (mg/L) & $0.32(0.14,0.60)$ & $0.27(0.22,0.35)$ & 0.792 \\
\hline CRP (mg/L) & $2.75(0.83,5.75)$ & $0.51(0.40,0.78)$ & 0.016 \\
\hline
\end{tabular}


Table 3 Circulating inflammatory markers between large- and small vessel disease in patients with acute ischemic stroke within I-3 days of onset.

\begin{tabular}{|c|c|c|c|}
\hline & $\begin{array}{l}\text { LAA } \\
(n=28)\end{array}$ & $\begin{array}{l}\text { SAO } \\
(n=29)\end{array}$ & Pvalue \\
\hline WBC $\left(\times 10^{9} / \mathrm{L}\right)$ & $6.8(5.8,7.6)$ & $6.6(5.7,7.8)$ & 0.703 \\
\hline Lymphocyte $\left(\times 10^{9} / \mathrm{L}\right)$ & $1.89(1.24,2.21)$ & $1.67(1.22,1.94)$ & 0.421 \\
\hline Monocyte $\left(\times 10^{9} / \mathrm{L}\right)$ & $0.44(0.37,0.59)$ & $0.53(0.43,0.67)$ & 0.261 \\
\hline CD40L (pg/ML) & $153.07(94.10,217.05)$ & $120.76(68.69,204.54)$ & 0.326 \\
\hline 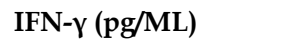 & $0(0,0)$ & $0(0,0)$ & 0.667 \\
\hline IL-1a (pg/ML) & $0(0,0)$ & $0(0,0)$ & 1.0 \\
\hline IL-1 $\beta$ (pg/ML) & $0(0,0)$ & $0(0,0)$ & 0.971 \\
\hline IL-6 (pg/ML) & $0.35(0,1.68)$ & $0.53(0.01,1.24)$ & 0.823 \\
\hline IL-8 (pg/ML) & $4.88(3.51,6.07)$ & $4.13(3.27,6.30)$ & 0.566 \\
\hline IL-17 (pg/ML) & $0(0,0)$ & $0(0,0)$ & 0.758 \\
\hline TNFa (pg/ML) & $7.50(4.75,8.44)$ & $6.52(5.44,9.67)$ & 0.961 \\
\hline $\mathrm{Fg}(\mathrm{g} / \mathrm{L})$ & $3.50(2.60,4.10)$ & $3.10(2.80,3.60)$ & 0.638 \\
\hline D-dimer $(\mathrm{mg} / \mathrm{L})$ & $0.29(0.18,0.63)$ & $0.26(0.18,0.53)$ & 0.875 \\
\hline CRP (mg/L) & $6.4(3.98,8.9)$ & $3.10(2.10,7.10)$ & 0.138 \\
\hline
\end{tabular}

Table 4 Influencing factors which were correlated with CRP level in patients with acute ischemic stroke.

\begin{tabular}{|c|c|c|c|c|c|}
\hline \multirow[t]{2}{*}{ variable } & \multicolumn{4}{|l|}{ Quartile of CRP } & \multirow[t]{2}{*}{ P Value } \\
\hline & $<2.1$ & 2.1-5.6 & $5.7-9.45$ & $>9.45$ & \\
\hline WBC & $5.58(5.50,6.75)$ & $6.60(4.88,10.13)$ & $6.75(5.85,9.15)$ & $7.25(6.20,7.93)$ & 0.404 \\
\hline Lymphocyte* & $2.17(1.58,2.76)$ & $1.64(1.41,1.92)$ & $1.92(1.30,2.22)$ & $1.49(0.44,2.76)$ & 0.030 \\
\hline Monocyte & $0.44(0.38,0.55)$ & $0.42(0.34,0.71)$ & $0.62(0.43,0.78)$ & $0.43(0.32,0.63)$ & 0.341 \\
\hline Cholesterol & $5.23(4.30,6.22)$ & $4.92(4.25,5.41)$ & $4.54(3.98,5.82)$ & $4.79(3.79,6.19)$ & 0.631 \\
\hline Triglyceride & $1.45(1.13,1.83)$ & $1.84(1.23,3.08)$ & $2.06(1.34,2.55)$ & $1.37(1.07,2.49)$ & 0.366 \\
\hline LDL & $3.16(2.86,4.46)$ & $2.96(2.73,3.21)$ & $2.49(2.22,3.55)$ & $2.55(2.13,4.06)$ & 0.257 \\
\hline HDL & $1.14(0.92,1.36)$ & $1.07(0.94,1.16)$ & $1.06(0.91,1.18)$ & $1.01(0.81,1.54)$ & 0.654 \\
\hline apoA & $1.20(1.05,1.48)$ & $1.17(1.05,1.46)$ & $1.24(1.15,1.40)$ & $1.15(0.98,1.25)$ & 0.451 \\
\hline аров & $1.10(0.92,1.22)$ & $1.08(0.91,1.30)$ & $1.04(0.74,1.20)$ & $1.10(0.80,1.15)$ & 0.777 \\
\hline Lp(a) & $0.13(0.07,0.26)$ & $0.20(0.13,0.25)$ & $0.15(0.06,0.30)$ & $0.13(0.09,0.27)$ & 0.614 \\
\hline CD40L (pg/ML) & $180.62(135.72,257.13)$ & $135.84(77.82,230.90)$ & $139.66(100.08,204.03)$ & $166.50(93.10,287.25)$ & 0.355 \\
\hline IFN- $\gamma(\mathrm{pg} / \mathrm{ML})$ & $0(0,0)$ & $0(0,0)$ & $0(0,0)$ & $0(0,0)$ & 0.804 \\
\hline IL-1a (pg/ML) & $0(0,0)$ & $0(0,0)$ & $0(0,0)$ & $0(0,0)$ & 1 \\
\hline IL-1 $\beta$ (pg/ML) & $0(0,0)$ & $0(0,0)$ & $0(0,0)$ & $0(0,0)$ & 0.484 \\
\hline IL-6 (pg/ML)* & $0.32(0.00,0.92)$ & $0.98(0.58,2.05)$ & $0.30(0.00,1.83)$ & $3.26(2.21,6.40)$ & $<0.001$ \\
\hline IL-8 (pg/ML) & $6.16(3.04,8.15)$ & $4.31(3.39,6.25)$ & $4.15(3.51,6.83)$ & $4.91(2.01,7.70)$ & 0.852 \\
\hline IL-17 (pg/ML) & $0(0,0)$ & $0(0,0)$ & $0(0,0)$ & $0(0,0)$ & 0.818 \\
\hline TNFa (pg/ML) & $5.87(4.90,8.28)$ & $7.58(5.53,9.65)$ & $5.91(3.82,8.53)$ & $7.29(4.62,14.56)$ & 0.302 \\
\hline $\mathrm{Fg}(\mathrm{g} / \mathrm{L})^{*}$ & $0.30(2.13,3.43)$ & $3.40(2.45,4.10)$ & $3.20(2.25,3.55)$ & $3.80(3.45,4.75)$ & 0.017 \\
\hline D-dimer $(\mathrm{mg} / \mathrm{L})^{*}$ & $0.27(0.19,0.35)$ & $0.20(0.13,0.38)$ & $0.32(0.16,0.71)$ & $0.56(0.35,0.92)$ & 0.009 \\
\hline NIHSS & $3.5(1,5)$ & $5(2,10)$ & $3(1.25,7.75)$ & $8(3,11)$ & 0.095 \\
\hline Infact volume & $0.90(0.13,11.66)$ & $3.30(1.23,11.66)$ & $2.14(0.34,2.52)$ & $34.65(23.34,254.83)$ & $<0.001$ \\
\hline
\end{tabular}

Table 5 Circulating inflammatory markers between smaller large stroke and small vessel disease in patients with acute ischemic stroke (infarct volume $1.3-1.82 \mathrm{~cm}^{3}$ )

\begin{tabular}{|c|c|c|c|}
\hline & $\begin{array}{l}\text { LAA } \\
(n=23)\end{array}$ & $\begin{array}{c}\text { SAO } \\
(n=15)\end{array}$ & P value \\
\hline WBC $\left(\times 10^{9} / \mathrm{L}\right)$ & $5.8(5.7,7.2)$ & $6.3(5.8,8.88)$ & 0.808 \\
\hline Lymphocyte $\left(\times 10^{9} / \mathrm{L}\right)$ & $1.8(1.37,2.08)$ & $1.93(1.41,3.24)$ & 0.423 \\
\hline Monocyte $\left(\times 10^{9} / \mathrm{L}\right)$ & $0.44(0.40,0.58)$ & $0.54(0.51,0.88)$ & 0.225 \\
\hline CD40L (pg/ML) & $168.30(104.52,312.12)$ & $82.55(36.60,120.92)$ & 0.683 \\
\hline IFN- $\gamma(\mathrm{pg} / \mathrm{ML})$ & $0(0,0)$ & $0(0,0)$ & 0.947 \\
\hline IL-1a (pg/ML) & $0(0,0)$ & $0(0,0)$ & 1.0 \\
\hline IL-1 $\beta$ (pg/ML) & $0(0,0)$ & $0(0,0)$ & 0.633 \\
\hline IL-6 (pg/ML) & $0.26(0.03,2.61)$ & $0.5(0,2.26)$ & 0.303 \\
\hline IL-8 (pg/ML) & $6.25(3.74,8.05)$ & $3.33(2.42,3.90)$ & 0.597 \\
\hline
\end{tabular}




\begin{tabular}{llll}
\hline IL-17 (pg/ML) & $0(0,0)$ & $0(0,0)$ & 0.270 \\
TNFa (pg/ML) & $6.65(4.68,9.31)$ & $5.40(4.19,8.25)$ & 0.549 \\
Fg (g/L) & $3.50(2.65,3.85)$ & $2.95(2.40,3.48)$ & 0.495 \\
D-dimer (mg/L) & $0.34(0.13,0.59)$ & $0.25(0.19,0.57)$ & 0.817 \\
CRP (mg/L) & $4.9(3.5,7.6)$ & $2.3(1.2,6.1)$ & 0.05 \\
\hline
\end{tabular}

\section{DISCUSSIONS}

Our study demonstrated that among a panel of inflammatory markers, CRP was the only one, which can distinguish large- from small vessel disease in acute ischemia stroke patients with $85.7 \%$ sensitivity. Rest cytokines such as CD40L, IFN- $\gamma$, IL-1 $\alpha$, IL-1 $\beta$, IL-6, IL-8, IL-17 and TNF-a etc, serum lipid and fibriolytic/coagulation were not sensitive for the etiological diagnosis in non-cardioembolic stroke. Moreover, IL-6, lymphocyte, Fibrinogen and D-dimer were correlated with the plasma level of CRP.

Atherosclerosis is the main basic pathophysiology in patients with non-cardioembolic stroke including large and small vessel disease. The metabolism of lipid and chronic inflammation are involved in the occurrence and progression of atherosclerosis. LDL is deposited in the lining of the arteries and forms a plaque. When the plaque blocked the vessel or instability, the acute ischemic stroke is induced and subsequently the inflammatory damage is initiated in the core and peripheral ischemia area in the brain. The coagulation and fibrinolytic procedure are also trigged by the thrombus. In general, inflammatory response plays an important role in patients with LAA and SAO, associated with instability of plaque, stenosis or occlusion of the large or small vessels, and the secondary ischemic cerebral damage. Many studies demonstrated that high levels of inflammatory mediator such as plasma IL-6, TNF, IL-1, CRP etc. could predict stroke severity and poor long-term outcome. We hypothesized that inflammatory makers may have a potential to distinguish occurrence of LAA or SAO. Hence, we choose a panel of blood biomarkers such as pro-inflammatory cytokine commonly studied, fibrinolytic/coagulation mediators, serum lipid and CRP to measure, and attempted to determinate non-cardioembolic stroke subtype. It is interesting that pro-inflammatory cytokines, fibrinolytic/coagulation mediators, and serum lipid were all useless to confirm non-cardioembolic stroke subtype except CRP. The LAA subtype showed a higher CRP level in plasma compared to SAO. This result suggested that CRP possibly play an important role in the instability and gravity of plaque, compared to the other cytokines, the lipid factors, etc. Interestingly, our data showed that pro-inflammatory cytokine "IL-6" and fibrinolytic/coagulative maker "fibrino- gen" "D-dimer" was related with CRP levels. It provides additional evidence that only CRP could differ LAA and ASO among a panel of markers. CRP could possibly represent that multiple pathway including inflammatory response, oxidant and fibrinolytic procedure as a marker of combine action. Moreover, our result showed that smaller large vessel stroke had a higher CRP level than SAO $(p=0.05)$. We believe a larger amount of patient number may increase the significant difference. CRP may be applied clinically in cases when determining subtype based solely on clinical presentation is difficult.

CRP was an acute-phase protein, which was synthesized and released into the peripheral blood by the liver in response to inflammation. Normally, CRP level in plasma is very low. When the acute inflammatory response or tissue injury occurred, its level rapidly increased within several hours and reached a peak after 2 days of stroke. Data demonstrated that elevated CRP in plasma could predict not only future vascular events in healthy individuals, but also an increased risk of fatal or nonfatal cerebrovascular events in ischemic stroke patients [12]. Elevated CRP levels at admission are significantly associated with early neurologic deterioration [13], poorer functional outcome, both short- and long-term mortality after stroke [14-15] and predict a larger infarct size [16-17]. Our study provides further evidence that elevated CRP could be served as a biomarker for the diagnosis of non-cardioembolic stroke subtype, especially within 1 day of stroke onset. A higher level of CRP more than 3.2 is associated with LAA subtype, compared to SAO subtype. Ladenvall et al. demonstrated the similar result that CRP levels differed between etiological subtypes of ischemic stroke both in the acute phase and at the 3-month follow-up. CRP at follow-up was associated with overall ischemic stroke and the large-vessel disease subtype [18]. However, several different results had been also reported. It may exist some links between the LAA subtype of stroke and elevated oxLDL, but not CRP [19]. CRP is significantly associated with the risk of having a first ischemic stroke, especially for small-vessel disease [20]. The common limitation of two negative studies is a small number of LAA subtype, only 20 patients. It may result in different conclusion. It is limited that the specificity of CRP was not strong. The systemic inflammation, infection and atherosclerosis and several 
drugs e.g. statin [21] could influence its levels in plasma. Sometimes we must interpreter cautiously when the level of CRP in plasma was high. We should combine other examines such as blood test, history of the disease, clinical symptoms, history of medicaments, etc to analyze the results.

In summary, CRP is one of the most extensively studied markers, which was associated with all stages of atherosclerosis, from the formation of the plaque to the occurrence and the progression of acute ischemic stroke. Higher CRP level in LAA supported our hypothesis that LAA had a stronger activation of inflammation than SAO in the pathogenesis of stroke. Using the biomarker "CRP" may be a feasible strategy to improve the diagnosis of non-cardioembolic stroke subtype in the acute phase. Moreover, a decrease of CRP levels by several drugs such as statin and antiplatelet medication, would be accelerated for the application of optimal secondary prevention to get a better clinical outcome and a decrease rate of recidivation. A large population study was needed to confirm the predicted power of CRP for stroke subtype.

\section{Acknowledgement}

This study is supported by 973 Program of NBRP, China (2011CB504405, GYY, YW), NSFC (81200943, LZ), the Shanghai medical association (SHNR-003, LZ), Shanghai healthy bureau (20124217, LZ) and by the Science and Technology Commission of Shanghai Municipality (09140902400, GYY and 09ZR1415300, YW).

\section{Competing Interests}

The authors have declared that no competing interest exists.

\section{References}

1. Muir KW, Tyrrell P, Sattar N, Warburton E. Inflammation and ischaemic stroke. Curr Opin Neurol. 2007; 20: 334-42. doi:10.1097/WCO.0b013e32813ba15100019052-200706000-00016 [pii].

2. Lambertsen KL, Biber K, Finsen B. Inflammatory cytokines in experimental and human stroke. J Cereb Blood Flow Metab. 2012; 32: 1677-98. doi:jcbfm201288 [pii]10.1038/jcbfm.2012.88.

3. Tuttolomondo A, Di Raimondo D, di Sciacca R, Pinto A, Licata G. Inflammatory cytokines in acute ischemic stroke. Curr Pharm Des. 2008; 14: 3574-89.

4. Basic Kes V, Simundic AM, Nikolac N, Topic E, Demarin V. Pro-inflammatory and anti-inflammatory cytokines in acute ischemic stroke and their relation to early neurological deficit and stroke outcome. Clin Biochem. 2008; 41: 1330-4. doi:S0009-9120(08)00379-2 [pii]10.1016/j.clinbiochem.2008.08.080.

5. Tuttolomondo A, Di Raimondo D, Pecoraro R, Arnao V, Pinto A, Licata G. Inflammation in ischemic stroke subtypes. Curr Pharm Des. 2012; 18 : 4289-310. doi:CPD-EPUB-20120229-002 [pii].

6. Beer C, Blacker D, Hankey GJ, Puddey IB. Association of clinical and aetiologic subtype of acute ischaemic stroke with inflammation, oxidative stress and vascular function: a cross-sectional observational study. Med Sci Monit. 2011; 17: CR467-73. doi:881931 [pii].

7. Rao R, Tah V, Casas JP, Hingorani A, Whittaker J, Smeeth L, et al. Ischaemic stroke subtypes and their genetic basis: a comprehensive meta-analysis of small and large vessel stroke. Eur Neurol. 2009; 61: 76-86. doi:000177939 [pii]10.1159/000177939.
8. Brisset M, Boutouyrie P, Pico F, Zhu Y, Zureik M, Schilling S, et al. Large-vessel correlates of cerebral small-vessel disease. Neurology. 2013; 80: 662-9. doi:WNL.0b013e318281ccc2[pii]10.1212/WNL. 0b013e318281ccc2.

9. Jung KW, Shon YM, Yang DW, Kim BS, Cho AH. Coexisting carotid atherosclerosis in patients with intracranial small- or large-vessel disease. J Clin Neurol. 2012; 8: 104-8. doi:10.3988/jcn.2012.8.2.104.

10. Adams HP, Jr., Bendixen BH, Kappelle LJ, Biller J, Love BB, Gordon DL, et al. Classification of subtype of acute ischemic stroke. Definitions for use in a multicenter clinical trial. TOAST. Trial of Org 10172 in Acute Stroke Treatment. Stroke. 1993; 24: 35-41.

11. Sims JR, Gharai LR, Schaefer PW, Vangel M, Rosenthal ES, Lev MH, et al. $\mathrm{ABC} / 2$ for rapid clinical estimate of infarct, perfusion, and mismatch volumes. Neurology. 2009; 72: 2104-10. doi:72/24/2104 [pii]10.1212/WNL.0b013e3181aa5329.

12. Di Napoli M, Schwaninger M, Cappelli R, Ceccarelli E, Di Gianfilippo G, Donati $\mathrm{C}$, et al. Evaluation of C-reactive protein measurement for assessing the risk and prognosis in ischemic stroke: a statement for health care professionals from the CRP Pooling Project members. Stroke, a journal of cerebral circulation. 2005; 36: 1316-29. doi:10.1161/01.STR.0000165929.78756.ed.

13. Seo WK, Seok HY, Kim JH, Park MH, Yu SW, Oh K, et al. C-Reactive protein is a predictor of early neurologic deterioration in acute ischemic stroke. Journal of stroke and cerebrovascular diseases : the official journal of National Stroke Association. 2012; 21: 181-6. doi:10.1016/j.jstrokecerebrovasdis.2010.06.002.

14. Chan CP, Jiang HL, Leung LY, Wan WM, Cheng NM, Ip WS, et al. Multiple atherosclerosis-related biomarkers associated with short- and long-term mortality after stroke. Clinical biochemistry. 2012; 45: 1308-15. doi:10.1016/j.clinbiochem.2012.06.014.

15. Dewan KR, Rana PV. C-reactive protein and early mortality in acute ischemic stroke. Kathmandu Univ Med J (KUMJ). 2011; 9: 252-5.

16. Hamidon BB, Sapiah S, Nawawi H, Raymond AA. The prognostic value of C-reactive protein (CRP) levels in patients with acute ischaemic stroke. The Medical journal of Malaysia. 2004; 59: 631-7.

17. Yu Q, Lin $Y$, Yang $P$, Wang $Y$, Zhao S, Fan J, et al. C-reactive protein is associated with the progression of acute embolic stroke in rabbit model. Journal of thrombosis and thrombolysis. 2012; 33: 301-7. doi:10.1007/s11239-011-0627-0.

18. Ladenvall C, Jood K, Blomstrand C, Nilsson S, Jern C, Ladenvall P. Serum C-reactive protein concentration and genotype in relation to ischemic stroke subtype. Stroke, a journal of cerebral circulation. 2006; 37: 2018-23. doi:10.1161/01.STR.0000231872.86071.68.

19. Vibo R, Korv J, Roose M, Kampus P, Muda P, Zilmer K, et al. Acute phase proteins and oxidised low-density lipoprotein in association with ischemic stroke subtype, severity and outcome. Free radical research. 2007; 41: 282-7. doi:10.1080/10715760601083235.

20. Andersson J, Johansson L, Ladenvall P, Wiklund PG, Stegmayr B, Jern C, et al. C-reactive protein is a determinant of first-ever stroke: prospective nested case-referent study. Cerebrovasc Dis. 2009; 27: 544-51. doi:10.1159/000214217.

21. Krupinski J, Turu MM, Slevin M, Martinez-Gonzalez J. Carotid plaque, stroke pathogenesis, and CRP: treatment of ischemic stroke. Current treatment options in cardiovascular medicine. 2007; 9: 229-35. 